\title{
Long-term results of the GSB III elbow arthroplasty
}

\author{
N. Gschwend, N. H. Scheier, A. R. Baehler \\ From the Schulthess Clinic, Zürich, Switzerland
}

$\mathbf{B}^{\mathrm{e}}$ etween 1978 and 1986, 59 patients received a GSB III elbow prosthesis, six of them in both elbows. Rheumatoid arthritis (RA) was the underlying cause in 51 of the patients and post-traumatic osteoarthritis (PTOA) in eight. Of these, 24 patients ( 28 prostheses) have since died; two, both operated on bilaterally, had had their implants for more than ten years and had already been assessed for inclusion in the long-term follow-up. Two patients, each with one elbow prosthesis, have been lost to follow-up and three males who are still living (two with PTOA, one with juvenile RA) had their prosthesis removed before ten years had elapsed.

The remaining 32 patients (28 RA, 4 PTOA) with 36 GSB III elbows were examined clinically and radiologically after a mean period of $\mathbf{1 3 . 5}$ years. Pain was considerably reduced in $91.6 \%$. Mobility was increased by $37^{\circ}$ in those with $\mathrm{RA}$ and by $67^{\circ}$ in those with PTOA.

There were three cases of aseptic loosening and three of deep infection. The main complication was disassembly of the prosthetic component in nine elbows (13.8\%). This last group included two patients with postoperative fractures unrelated to the operative technique and one with neuropathic arthritis. Ulnar neuritis occurred in two patients.

Since $87.7 \%$ of all the GSB III prostheses implanted in this period remained in situ, our results are comparable with those for hip and knee arthroplasty.

J Bone Joint Surg [Br] 1999;81-B:1005-12.

Received 16 September 1998; Accepted after revision 23 April 1999

The outcome of every artificial joint replacement is evaluated for pain relief and functional improvement and also for the duration of this success. The last factor is critical

N. Gschwend, MD, Professor of Orthopaedics

N. H. Scheier, MD, Professor of Orthopaedics

Schulthess Clinic, Lengghalde 2, CH-8008 Zürich, Switzerland.

A. R. Baehler

Engineering, Kapfsteig 44 A, CH-8032 Zürich, Switzerland.

Correspondence should be sent to Professor N. Gschwend.

(C)1999 British Editorial Society of Bone and Joint Surgery 0301-620X/99/69495\$2.00

VOL. 81-B, No. 6, NOVEMBER 1999 since more young patients undergo joint replacement and revision surgery, particularly with the exchange of a prosthesis, has many technical problems with a reduced prospect of success.

Replacement arthroplasty of the elbow is not a common operation. The number has increased in the last ten years, and the results are improving, but the percentage of complications and revisions reported in the literature is higher than for the hip, knee and shoulder. An analysis of the most relevant literature on elbow arthroplasty published between 1987 and $1997^{1-35}$ shows a mean period of follow-up of 4.9 years $(0.25$ to 17$)$ and few of these studies had uninterrupted and complete series.

Our aim was to analyse the long-term results of a consecutive series of patients with the GSB III elbow prostheses implanted for more than ten years.

\section{Patients and Methods}

The requirements of a good elbow prosthesis are: 1) optimum adaptation of the implant to the physiological requirements; 2) unobstructed flexion and extension; 3) lateral stability; 4) construction from biomechanically accepted materials; and 5) minimum bone resection to enable greater choice if revision is needed.

Our experience with the GSB I elbow prosthesis began in 1971. At that time all rigid hinge joints showed a high rate of loosening. As a result of an analysis of their mode of failure $^{36}$ (Figs 1a to 1c) the GSB III elbow prosthesis was developed (Allo Pro AG, Baar, Switzerland). It has essentially remained unchanged and has been in clinical use since 1978. The humeral component of the implant is adapted as closely as possible to the normal anatomy. It has large surfaces for support on the condyles and a wide stem for transference of rotational stress. The oval, loose-link connection between the ulna and humerus allows an angle of $5^{\circ}$ for abduction and adduction and rotational movement. All articulating surfaces are coated with polyethylene, including the peg in the rotation casing of the humeral section and the ulnar peg within the hinge casing. The 'plug-in' non-constrained clearance of the ulnar component and the mobile rotation casing give flexible stability.

The extensive surface support from the humeral condyles and the broad intramedullary stems help to distribute loads 


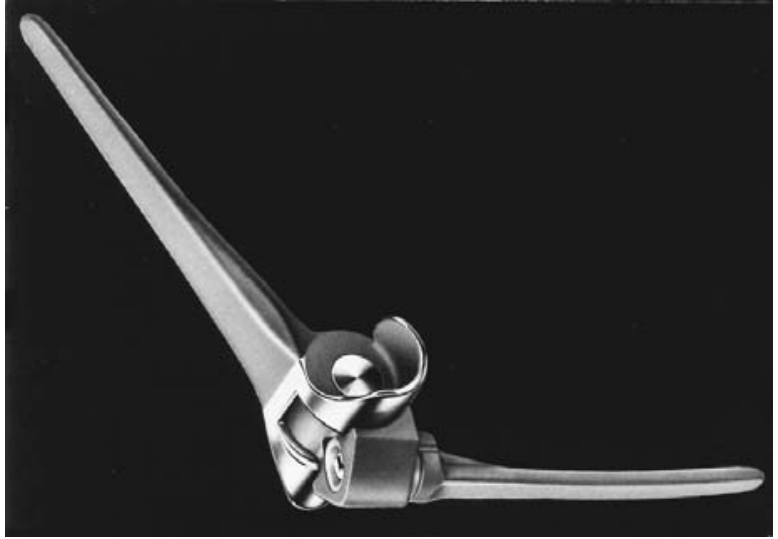

Fig. 1a

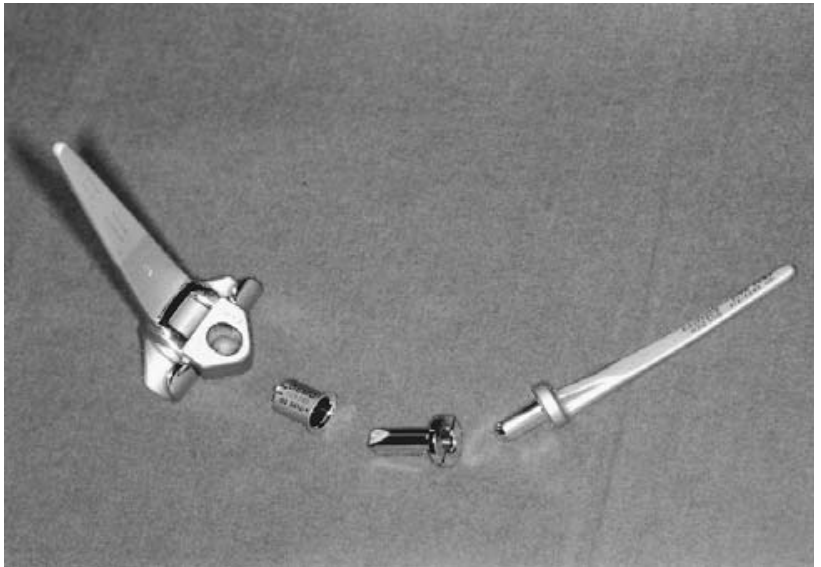

Fig. 1c

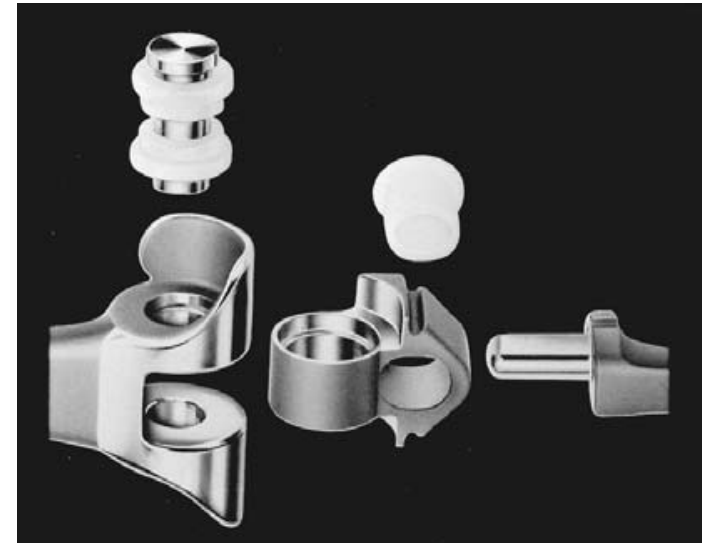

Fig. 1b

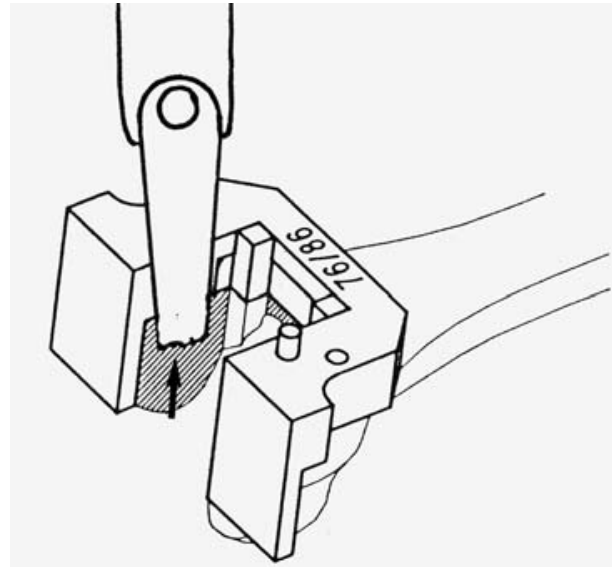

Fig. 1d

Figure 1a - The GSB III elbow prosthesis; a sloppy hinge with condylar flanges allows $5^{\circ}$ of loose movement between the parts Figure $1 \mathrm{~b}$ - The GSB III elbow prosthesis disassembled, with high-density polyethylene bushes for the hinge and the coupling extension. Figure 1c - Lengthening device for the ulnar component in case of disassembly. Figure 1d - Guide for resection of the intercondylar bone bloc. The arrow points to the centre of rotation.

evenly. The clearance between the humerus and the ulna ameliorates even strong traction forces, such as those created during heavy lifting. The capsular and ligamentous apparatus contributes to stability. ${ }^{37,38}$ Thus, the two main causes of loosening are greatly reduced. The artificial joint theoretically allows hyperextension of approximately $10^{\circ}$ and has a range of movement through $180^{\circ}$.

The metal components which are exposed to torsion and flexion are constructed from Protasul-10 alloy resistant to fatigue-fracture $^{39}$ and the components which are potentially subject to movement fatigue are made of wear-resistant cast alloy Protasul-2. ${ }^{39}$ Both parts of each prosthesis are welded together. The polyethylene cases are made of Sulene-PE. ${ }^{40}$ The GSB III elbow prosthesis has three humeral sizes and four ulnar components all of which can be freely combined with each other.

Operative technique. The anaesthetised patient is placed in a lateral position on the table with the arm under tourniquet at a right angle on a padded support. A dorsal skin incision is made passing the olecranon on its radial side; a thick flap is raised towards the medial side exposing the triceps tendon. The ulnar nerve is then mobilised from proximal to distal as far as the first muscular branch distal to the sulcus. Care is taken not to injure the accompanying vessels. The triceps tendon is split centrally over a distance of $7 \mathrm{~cm}$ and elevated, with a thin layer of bone from the olecranon (Fig. 2). The medial side of the humeroulnar joint is exposed and the sharp bony edge near the insertion of the ventral portion of the ulnar collateral ligament is removed. This is necessary to avoid damaging the ulnar nerve which lies close by. The radiohumeral and radioulnar joints are then exposed.

Precision instruments, including guides, templates and reamers, are used to fashion the humerus to seat its component at the centre of rotation of the elbow (Fig. 1d). The ulna is similarly prepared with special instruments. After a trial fit, both components are cemented into place and assembled. The extensor mechanism is then replaced over the olecranon and secured by non-absorbable sutures (Fig. 2b). The elbow is supported in a plaster slab at $40^{\circ}$ flexion for ten days. Passive flexion exercises using a continuouspassive-motion splint are started for five days but should 


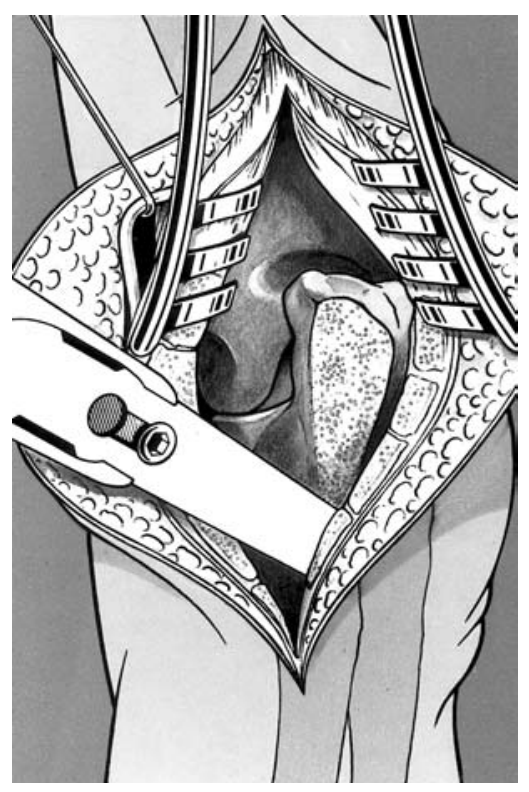

Fig. 2a

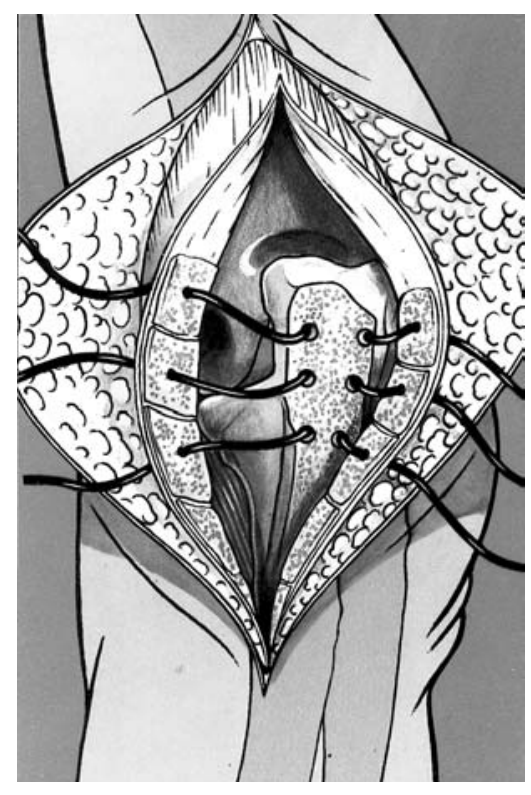

Fig. $2 b$

Diagrams of the operative technique showing the transtricipital approach a) and the final sutures b).

not exceed $90^{\circ}$ in the first ten days. Thereafter a gradual increase in active flexion and full passive extension are allowed. The patient remains in hospital for a week and rehabilitation continues on an outpatient basis.

We performed a retrospective clinical and radiological examination of 59 patients (65 elbows) who had received a GSB III elbow prosthesis between 1978 and 1986. At the time of the final follow-up, 24 patients (28 elbows) had died from unrelated causes but two of these (four elbows) had already survived for more than ten years and had been evaluated for inclusion in the long-term analysis. In addition, three had had their prostheses removed before ten years had elapsed, in two with post-traumatic osteoarthritis (PTOA) because of recurrent dislocation and in one with juvenile chronic arthritis because of disassembly and secondary deep infection. They are included in the complications. All patients who died before ten years had elapsed are also included in the discussion of the complications and revisions. Two patients, each with one prosthesis, could not be traced since they had emigrated a few years after the operation. At their last examination, one and five years following surgery, respectively, the results were clinically and radiologically excellent. The long-term results, concerning pain, mobility and radiological appearance, relate to 32 patients with 36 prostheses. The reported complications concern all 65 prostheses implanted between 1978 and 1986. The surgery was performed by the senior author (NG) in 24 patients and by a few other experienced surgeons in the remaining 12 .

The mean follow-up of the 32 patients was for 13.5 years (10 to 20); 28 (22 women, 6 men) had rheumatoid arthritis (RA) and four PTOA ( 2 women, 2 men). The mean age of all the patients at the time of operation was 45.7 years $(21$ to 71); that of the female RA patients was 50.0 years (21 to 71 ), of the male RA patients 47.3 years (35 to 69), of the female PTOA patients 47.5 years and of the male PTOA patients 38.5 years. Of the 36 prostheses, 22 were on the right side and 14 on the left. Contingency analyses were used for pre- and postoperative pain and changes in the range of movement were assessed by Student's $t$-test.

\section{Results}

Figure 3 shows four cases of GSB III elbow arthroplasty in which the follow-up was for 11, 13, 15 and 18 years, respectively.

Pain. This was graded as: 1) none or only occasional slight pain, not requiring medication; 2) moderate more or less constant pain, eventually requiring medication; and 3 ) intolerable pain (Table I).

Before operation, $77.7 \%$ of the patients complained of intolerable pain (grade 3) and this was the indication for surgery. Afterwards, $91.6 \%$ had none or only occasional slight pain. This difference was highly significant $(\mathrm{p}<0.0001)$. The three patients who complained of pain after operation (grades 2 and 3) had RA and in two of them there were signs of loosening of the prosthesis.

Range of movement. The improvement in the range of movement is shown in Table II. In the RA group the mean increase in movement was $37^{\circ}$; in the PTOA group it was $67^{\circ}$ ( $p<0.0001$ for each group). The mean point of maximum flexion achieved after operation was $144^{\circ}$ for the RA group and $141^{\circ}$ for the PTOA group. The extension deficit was $28^{\circ}$ in the RA group and $44^{\circ}$ in the PTOA group.

Complications. The three main complications were aseptic loosening, infection and disassembly. 

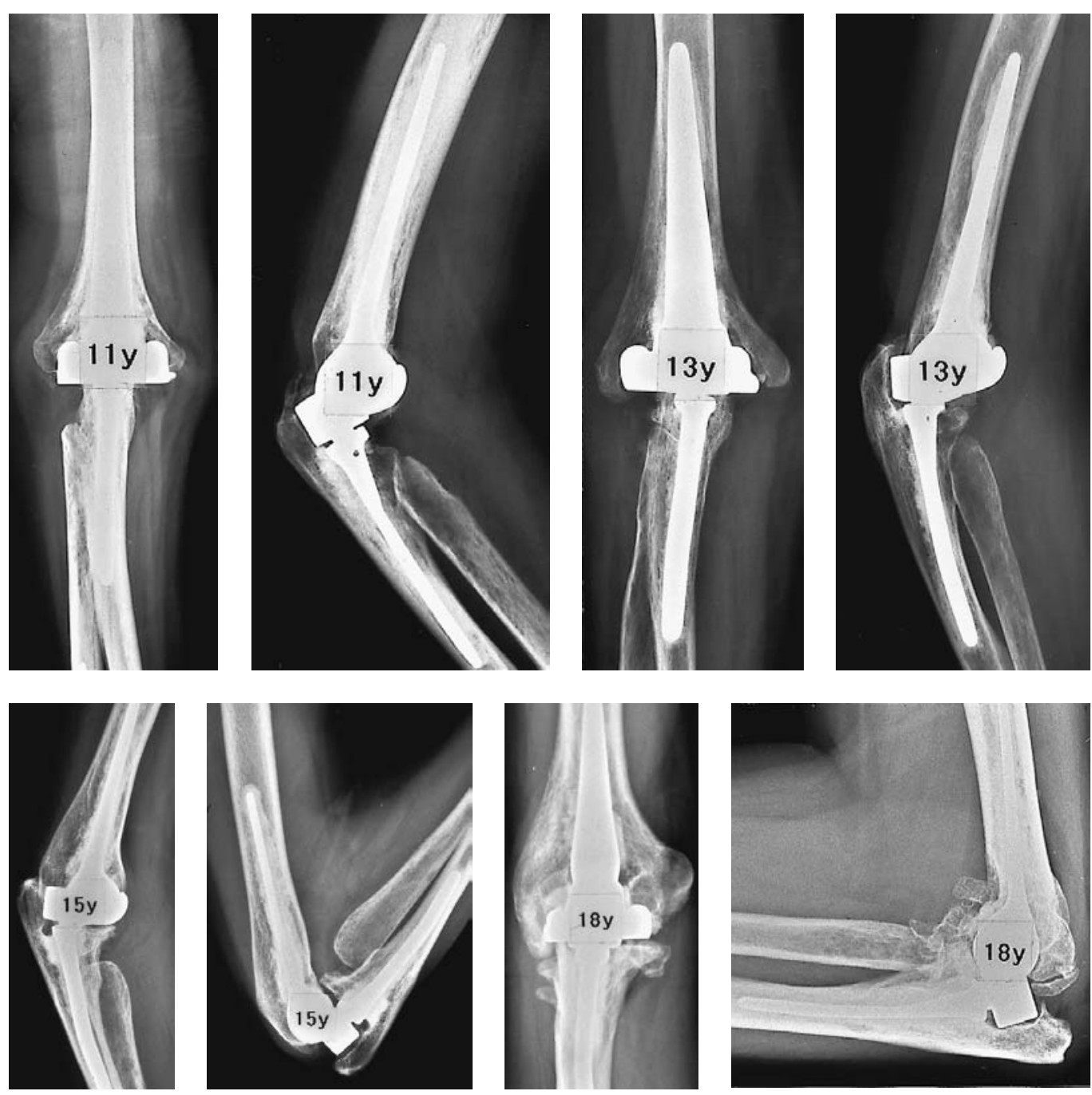

Fig. 3

AP and lateral views of four cases with a follow-up for 11, 13, 15 and 18 years, respectively.

Aseptic loosening. Of the 65 prostheses implanted, aseptic loosening was identified in three $(4.6 \%)$. Two of these patients had been followed for more than ten years. In one, a 72-year-old woman with RA, there was insufficient

Table I. Details of the amount of pain before and after operation, by number and percentage

\begin{tabular}{lcc}
\hline Grade* & Before operation & After operation \\
\hline 1 & $4(11.1)$ & $33(91.6)$ \\
2 & $4(11.1)$ & $2(5.5)$ \\
3 & $28(77.7)$ & $1(2.7)$ \\
\hline
\end{tabular}

* see text cement. The humeral component was exchanged 11 years after the first operation and recemented with a satisfactory result.

The second patient was an 84-year-old woman with RA who had had a GSB III prosthesis on each side. That on the left was still secure and painless after 15 years. The range of movement was from $50^{\circ}$ to $135^{\circ}$. By contrast, on the right side, which had been operated on 14 years before, the surgeon had perforated the shaft of the ulna, despite which the artificial joint functioned well. The patient, however, had later to undergo bilateral knee and hip replacements and also revision operations on a hip and a knee. One

Table II. Mean (SD; range) range of movement (degrees) before and after operation in the RA and PTOA groups

\begin{tabular}{|c|c|c|c|c|c|c|}
\hline & \multicolumn{3}{|l|}{ Before operation } & \multicolumn{3}{|l|}{ After operation } \\
\hline & $\mathbf{R A}$ & PTOA & $p$ value & $\mathbf{R A}$ & PTOA & p value \\
\hline FFD* & $40.3(20.9 ; 0$ to 95$)$ & $58(21.7 ; 30$ to 80$)$ & & $27.8(17.4 ; 5$ to 85$)$ & $44(18.5 ; 25$ to 70$)$ & \\
\hline Flexion & $120.0(21.2 ; 35$ to 150$)$ & $88.0(22.8 ; 50$ to 110$)$ & & $144.2(8.5 ; 130$ to 160$)$ & $141.0(7.4 ; 130$ to 150$)$ & \\
\hline Total & $79.7(31.2 ; 0$ to 130$)$ & $30.0(12.2 ; 20$ to 50$)$ & $<0.0001$ & $116.3(21.5 ; 55$ to 145$)$ & $97.0(20.5 ; 70$ to 120$)$ & $<0.0001$ \\
\hline
\end{tabular}

* fixed flexion deformity or loss of extension 
shoulder was also replaced. These operations and her advanced age necessitated the use of elbow crutches. She then fell heavily on her right elbow and complained of pain ever since. All of these factors contributed to aseptic loosening of both components 14 years after the initial operation. The patient's poor general condition and advanced age contraindicated revision surgery and she has since died.

The third case of aseptic loosening was found in a 56-year-old woman with RA. Unusually delicate bones required that the prosthesis be modified and the ulnar stem shortened. A complete envelope of radiotranslucency developed at three years with some scalloping of the ulna. The patient did not complain of pain and had a good functional result until she died at the age of 64 years.

Infection. This occurred in four of the 65 elbows (6\%). One superficial infection in a 63-year-old woman with RA healed after treatment with antibiotics. Three deep infections required removal of the prosthesis, which was the usual procedure in the earlier years, and the elbow was left as an excision. All three infections followed revision surgery; two of them are also mentioned among the disassemblies. There were no deep infections in the 36 cases with a follow-up of more than ten years.

The first deep infection occurred in a 70-year-old woman with RA. She sustained a fracture of the ulnar condyle two years after elbow arthroplasty. Osteosynthesis and a consequent disassembly required several further operations (vide infra). The second was in a 61-year-old woman with RA who suffered a fracture of the ulnar condyle during the initial arthroplasty. The bone was fixed by Kirschner wires and a screw, but problems with wound healing and necrosis of the triceps tendon necessitated a radical revision three weeks later. An infection developed and the prosthesis was removed. These two patients died within ten years of implantation of the GSB III arthroplasty.

The third infection occurred in a 32-year-old man with severe juvenile arthritis. The hinge joint of the prosthesis locked spontaneously in extension and disassembly of both components occurred. Revision surgery led to an infection and the prosthesis had to be removed (vide infra).

Disassembly of prosthetic components. Disassembly of the prosthetic components was a fairly common complication in our early series and occurred in nine cases (13.6\%). There were several causes, including inexact placement of the humeral component at the centre of rotation of the natural elbow and too extensive release of soft tissue. Two male patients, aged 33 and 38 years with PTOA, were engaged in heavy physical work. In one the prosthesis was removed leaving him with an excision arthroplasty, and in the other arthrodesis was performed, the only example of that operation in the series. The first patient was unhappy and a further revision replacement arthroplasty was carried out. This remains satisfactory seven years later.

A 79-year-old woman with RA suffered disassembly three years after arthroplasty following a fracture of the olecranon which failed to unite. After repair and recon- struction, the elbow has remained stable. The patient has since died from an unrelated cause.

Another 70-year-old woman with RA sustained a fracture of the ulnar condyle two years after surgery. Disassembly occurred necessitating revision surgery. Infection complicated this case and the prosthesis was removed (vide supra).

In a 32-year-old man, suffering from severe juvenile arthritis, the GSB hinge became locked in extension causing disassembly of the ulnar component when he attempted to flex the elbow. Revision also led to infection and removal of the prosthesis (vide supra).

In a 68-year-old woman with RA both elbows were replaced within two years of each other. After 18 years the hinge of the first elbow replacement locked because the components disassembled. The humeral component was exchanged for a locked hinge mechanism although it showed no signs of loosening. The result was classified as good clinically and radiologically.

A 46-year-old woman with RA developed syringomyelia with severe weakness of the triceps and bilateral dislocation of the shoulders. Disassembly of the elbow prosthesis followed. After reassembling both components the patient was satisfied, except for a considerable fixed flexion deformity. She died nine years later.

A 60-year-old woman with RA had removal of a loose GSB I prosthesis which had been implanted in the early 1970s. She was not satisfied with the excision arthroplasty and requested a repeat replacement. A new GSB III prosthesis disassembled six years after surgery, probably due to a slack triceps. We reassembled both components and the prosthesis worked well until her death four years after the last operation.

The last patient was a very active 42-year-old woman with RA. During strenuous activity, she disassembled the prosthesis because the hinge mechanism locked in extension, apparently because it did not have enough clearance. After the well-fixed humeral component had been exchanged, the elbow functioned well with a range of active flexion of $20^{\circ}$ to $135^{\circ}$.

Several other revision procedures were required. A disrupted triceps was repaired by a secondary reefing in a 40-year-old woman with PTOA. A 53-year-old woman with RA had extension lengthening of the biceps and brachialis to improve an extensor deficit of $85^{\circ}$. Little gain was achieved. Both patients are free from pain and are satisfied with their function despite the major deficit in extension. The interfaces looked normal at 15 and 16 years, respectively, after surgery.

In a 68-year-old woman with RA, the humerus cracked during insertion and a displaced fracture of the ulnar condyle occurred at two weeks, requiring fixation with a small plate and screws. The result is clinically and radiologically good at 12 years except for a slight hyperaesthesia in the area of the ulnar nerve.

Postoperative hyperaesthesia occurred in the distribution 
of the ulnar nerve in two cases, both for minor revision, one to remove a sharp exostosis at the olecranon and another for bursitis of the olecranon.

In summary, eight GSB III prostheses from a total of 65 implanted between 1978 and 1986, had to be removed or changed $(12.3 \%)$. Three were removed for deep infection (4.6\%), one for aseptic loosening (1.5\%) and one for recurrent disassembly (1.5\%). In three cases of disassembly $(4.6 \%)$ the humeral component was replaced. All these patients are satisfied with the result. Two patients with aseptic loosening (vide supra) have not been reoperated on. One was an 80-year-old woman who lived in a nursing home and died soon after, and the other had no pain despite complete radiolucency, but without migration; she died three years later.

\section{Discussion}

Artificial replacement of the elbow is not a common procedure. According to a meta-analysis of the world literature between 1986 and $1992,{ }^{41}$ the incidence of complications was $43 \%$ (357 of 828 implanted prostheses). In view of the high rate of revision of $18 \%(151 / 828)$ after a mean follow-up of 55 months, it is perhaps not surprising that there are still reservations concerning elbow arthroplasty. In the last few years, however, progress has been made with a diminishing number of complications. ${ }^{13,42-48}$

We have found only one report of the long-term results (>10 years) of the use of a sloppy hinge, ${ }^{49}$ the CoonradMorrey elbow prosthesis. It is the only sloppy hinge which, like ours, uses a flange on the anterior wall of the humerus. The authors analysed 69 patients with 78 elbow prostheses operated on between 1981 and 1986. The most recent follow-up was performed at the authors' institution in 36 elbows and by a questionnaire and examination by a local physician in 42 . Of the total, 46 elbows were followed for at least ten years. The mean duration of radiological followup was 9.6 years. The authors report a survivorship of $92.4 \%$ at ten years with 43 prostheses at risk; $13 \%$ had serious complications necessitating reoperation. They expressed some concern regarding the number of cases in which the high-density polyethylene bushes were worn $(7 \%$ completely, $8 \%$ partially) but were satisfied with the clinical results.

Another long-term study concerning the capitellocondylar prosthesis was presented to the AAOS meeting in 1999, with very good results at more than ten years (Ewald, personal communication). This is a non-linked prosthesis which requires good ligaments. It is mainly used in rheumatoid arthritis.

The GSB III prosthesis belongs, like the Coonrad-Morrey prosthesis, to the group of sloppy hinges which have a clearance between the humeral and ulnar components. It allows movements similar to those of the normal elbow laterally as well as longitudinally and in rotation, ${ }^{38}$ and therefore reduces the stresses on the interface. Unlike most other sloppy hinges, the GSB III prosthesis uses flanges resting on both condyles of the humerus. These distribute evenly the stresses acting on the extended elbow in a vertical direction and those on the flexed elbow in a dorsal direction, as well as in rotation. ${ }^{36}$ This is one of the main reasons for preserving both condyles or, if they are missing, for reconstructing them by autografts. ${ }^{50}$ The intrinsic stability provided by all linked prostheses, including the sloppy hinges, justifies extending the indication for artificial joint replacement to cases in which the ligamentous structures are defective and in which the articulating surfaces are more severely deformed or destroyed.

The series which we examined in our long-term followup was uninterrupted and two-thirds of the operations had been carried out by one surgeon. All had been followed up at our institution with the exception of two patients who had left the country relatively soon after the operation without indicating their whereabouts. All living recipients of the GSB III elbow prosthesis had a clinical and radiological examination at a mean of 13.5 years after surgery.

We also established the fate of the prosthesis in all patients who had died between 1978 and 1997. For this we used the last clinical and radiological data in our records. Two of the group of patients who had died had survived more than ten years after implantation of their prostheses, and their data were included in the long-term analysis. To obtain reliable information concerning the survivorship of the GSB III prosthesis, we felt it necessary to analyse all complications in both groups, including those who had died within ten years of the arthroplasty. We also included the eight patients with PTOA even although it is acknowledged that the results of elbow arthroplasty are better in RA patients in which the preoperative difficulties, as well as the physical stresses after surgery, are less than those in posttraumatic cases. Excluding the two young male patients with PTOA who had hard physical occupations, and whose disassembled prostheses required removal relatively soon after the initial operation, we found no major differences in the long-term results of the post-traumatic group compared with the RA group. The slightly greater loss of extension was probably due to the fact that six of the eight patients with PTOA had had previous surgery. The results concerning pain and range of movement of the 32 patients with 36 prostheses re-examined after more than ten years were satisfactory and were similar to our medium-term results published at an earlier date. ${ }^{51,52}$

Of special interest is the fact that we found only three cases of aseptic loosening, $4.6 \%$ of the whole series. This compares well with the meta-analysis of the world literature between 1986 and $1992^{41}$ which showed an incidence of radiological loosening of $17.2 \%$ (humerus $12.1 \%$, ulna $5.1 \%$ ) after a mean period of 55 months.

Our experience demonstrates the advantages of sloppy hinges in general and the GSB III prosthesis in particular. The cause of aseptic loosening in two of the three cases was insufficient cement covering of the stem, particularly 
the tip, and in the third case perforation of the ulna together with overloading of the prosthesis and a subsequent fall.

The rate of infection of $4.6 \%$ is also relatively low compared with $8.1 \%$ from the meta-analysis. ${ }^{41}$ In comparison with replacement arthroplasty of other joints, the rate of infection for the elbow is still too high. In our series, however, no cases followed a primary procedure; all occurred after one or more reoperations for complications. The likelihood of complications occurring at the elbow is greater than for other joints, because of the thin soft-tissue coverage, the length of the lever arms on either side of the joint and its exposure to external forces. Lesions of the ulnar nerve occurred in 3\% in our series, compared with $10.5 \%$ in the meta-analysis. We took particular care over the exposure of the ulnar nerve and its mobilisation without translocation in most cases.

In the early part of our experience with the prosthesis, we had encountered disassembly in $13.8 \%$ of our cases compared with $7.1 \%$ of instabilities and dislocations of unlinked elbow prostheses reported in the meta-analysis. The problem has now largely been resolved by the use of new instruments which allow more accurate location of the prosthesis with respect to the centre of the rotation of the normal elbow (Fig. 1d), and with the availability of an ulnar component which can be lengthened by means of a special device (Fig. 1c). Analysis of the causes of disassembly revealed unusual problems such as fracture of the olecranon or condyle, and insufficiency or paralysis of the triceps.

Although none of the authors have received or will receive benefits for personal or professional use from a commercial party related directly or indirectly to the subject of this article, benefits have been or will be received but are directed solely to a research fund, foundation, educational institution, or other non-profit institution with which one or more of the authors is associated.

\section{References}

1. Schneeberger AG, Adams R, Morrey BF. Semiconstrained total elbow replacement for the treatment of post-traumatic osteoarthrosis. $J$ Bone Joint Surg [Am] 1997;79-A:1211-22.

2. Cobb TK, Morrey BF. Total elbow arthroplasty as primary treatment for distal humeral fractures in elderly patients. J Bone Joint Surg [Am] 1997;79-A:826-32.

3. Risung F. The Norway elbow replacement: design, technique and results after nine years. J Bone Joint Surg [Br] 1997;79-B:394-402.

4. Andreassen G, Solheim LF. Follow-up of Souter elbow prostheses. Tidsskr Nor Laegeforen 1997;117:940-2.

5. King GJ, Adams RA, Morrey BF. Total elbow arthroplasty: revision with use of a non-custom semiconstrained prosthesis. J Bone Joint Surg [Am] 1997;79-A:394-400.

6. Chantelot C, Fontaine C, Migaud H, Duquennoy A. Complete elbow prosthesis for inflammatory and hemophiliac arthroplasty: a retrospective analysis of 22 cases. Ann Chir Main Memb Super 1997; 16:49-57.

7. Schemitsch EH, Ewald FC, Thornhill TS. Results of total elbow arthroplasty after excision of the radial head and synovectomy in patients who had rheumatoid arthritis. J Bone Joint Surg [Am] 1996; 78-A:1541-7.

8. Judet T, Garreau de Loubresse C, Piriou P, Charnley G. A floating prosthesis for radial head fractures. J Bone Joint Surg [Br] 1996;78-B: 244-9.

9. Ljung P, Jonsson K, Rydholm U. Short-term complications of the lateral approach for non-constrained elbow replacement: follow-up of 50 rheumatoid elbows. J Bone Joint Surg [Br] 1995;77-B:937-42.
10. Sjöden GO, Lundberg A, Blomgren H. Late results of the SouterStrathclyde total elbow prosthesis in rheumatoid arthritis. Acta Orthop Scand 1995;66:391-4.

11. Dent CM, Hoy G, Stanley JK. Revision of failed total elbow arthroplasty. J Bone Joint Surg [Br] 1995;77-B:691-5.

12. Morrey BF, Adams RA. Semiconstrained elbow replacement for distal humeral nonunion. J Bone Joint Surg [Br] 1995;77-B:67-72.

13. Kraay MJ, Figgie MP, Inglis AE, Wolfe SW, Ranawat CS. Primary semiconstrained total elbow arthroplasty: survival analysis of 113 consecutive cases. J Bone Joint Surg [Br] 1994;76-B:636-40.

14. Lyall HA, Cohen B, Clatworthy M, Constant CR. Results of the Souter-Strathclyde total elbow arthroplasty in patients with rheumatoid arthritis: a preliminary report. J Arthroplasty 1994;9:279-84.

15. Dhar S, Beddow FH. The modified Liverpool total elbow prosthesis. J Hand Surg Br 1994;19:224-8.

16. Brady O, Quinlan W. The Guildford elbow. J Hand Surg [Br] 1993; 18:389-93.

17. Ewald FC, Simmons ED, Sullivan JA, et al. Capitellocondylar total elbow replacement in rheumatoid arthritis: long-term results. $J$ Bone Joint Surg [Am] 1993;75-A:498-507.

18. Yatsuka T, Torisu T, Masuda Y, et al. The significance of total elbow replacement in the treatment of rheumatoid arthritis. Ryumachi 1993; 32:142-8.

19. Sjoden G, Blomgren G. The Souter-Strathclyde elbow replacement in rheumatoid arthritis: 13 patients followed for 5 (1-9) years. Acta Orthop Scand 1992;63:315-7.

20. Morrey BF, Adams RA. Semiconstrained arthroplasty for the treatment of rheumatoid arthritis of the elbow. J Bone Joint Surg [Am] 1992;74-A:479-90.

21. Ruth JT, Wilde AH. Capitellocondylar total elbow arthroplasty: a long-term follow-up study. J Bone Joint Surg [Am] 1992;74-A: 95-100.

22. Swanson AB, de Groot Swanson G, Masada K, et al. Constrained total elbow arthroplasty. J Arthroplasty 1991;6:203-12.

23. Pöll RG, Rozing PM. Use of the Souter-Strathclyde total elbow prosthesis in patients who have rheumatoid arthritis. J Bone Joint Surg [Am] 1991;73-A:1227-33.

24. Morrey BF, Adams R, Bryan RS. Total replacement for posttraumatic arthritis of the elbow. J Bone Joint Surg [Br] 1991;73-B: 607-12.

25. Hodgson SP, Parkinson RW, Noble J. Capitellocondylar total elbow replacement for rheumatoid arthritis. J R Coll Sur Edinb 1991;36: 133-5.

26. Trepman E, Vella IM, Ewald FC. Radial head replacement in capitellocondylar total elbow arthroplasty: 2- to 6-year follow-up evaluation in rheumatoid arthritis. J Arthroplasty 1991;6:67-77.

27. Burnett R, Fyfe IS. Souter-Strathclyde arthroplasty of the rheumatoid elbow: 23 cases followed for 3 years. Acta Orthop Scand 1991;62: 52-4.

28. Brumfield RH Jr, Kuschner SH, Gellman H, Redix L, Stevenson DV. Total elbow arthroplasty. J Arthroplasty 1990;5:359-63.

29. Jonsson B, Larsson SE. Elbow arthroplasty in rheumatoid arthritis: function after 1-2 years in 20 cases. Acta Orthop Scand 1990;61: 344-7.

30. Figgie MP, Inglis AE, Mow CS, et al. Results of reconstruction for failed total elbow arthroplasty. Clin Orthop 1990;253:123-32.

31. Kudo H, Iwano K. Total elbow arthroplasty with a non-constrained surface-replacement prosthesis in patients who have rheumatoid arthritis: a long-term follow-up study. J Bone Joint Surg [Am] 1990;72-A: $355-62$.

32. Weiland AJ, Weiss A-PC, Wills RP, Moore JR. Capitellocondylar total elbow replacement. J Bone Joint Surg [Am] 1989;71-A:217-22.

33. Friedman RJ, Lee DE, Ewald FC. Nonconstrained total elbow arthroplasty: development and results in patients with functional class IV rheumatoid arthritis. J Arthroplasty 1989;4:31-7.

34. Trancik T, Wilde AH, Borden LS. Capitellocondylar total elbow arthroplasty - two- to eight-year experience. Clin Orthop 1987;223: $175-80$.

35. Morrey BF, Bryan RS. Revision total elbow arthroplasty. $J$ Bone Joint Surg [Am] 1987;69-A:523-32.

36. Gschwend N. Surgical treatment of rheumatoid arthritis. Stuttgart: Thieme, 1980:61-5.

37. Herren DB, Schneeberger AG, Simmen BR, et al. Kinematics and laxity of the GSB III elbow prosthesis. Procs 7th Annual Conference of the European Orthopaedic Research Society, Barcelona, 1997. 
38. Herren DB, Simmen BR, Gschwend N, An KN. The role of the collateral ligaments in a semiconstrained total elbow arthroplasty (GSB III Elbow Prosthesis). Procs 7th Annual Conference of the European Orthopaedic Research Society, Barcelona, 1997.

39. Semlitsch M, Willert HG. The importance of remembering past mistakes when considering implant materials for future hip endoprostheses. In: Morscher EW, ed. Endoprosthetics. Berlin etc; Springer, 1995:18-33.

40. Streicher RM. Tribology of artificial joints. In: Morscher EW, ed. Endoprosthetics. Berlin etc; Springer, 1995:34-48.

41. Gschwend N, Simmen BR, Matejovsky Z. Late complications in elbow arthroplasty. J Shoulder Elbow Surg 1996;5,2/1:86-96.

42. Ewald FC. Capitellocondylar total elbow arthroplasty. In: Morrey, BF, ed. Master techniques in orthopaedic surgery: the elbow. New York: Raven Press Ltd, 1994.

43. Figgie HE, Inglis AE, Mow CS. A critical analysis of biomechanica factors affecting functional outcome in total elbow arthroplasty. $J$ Arthroplasty 1986;1:169-73.

44. Figgie HE III, Mow CS. Semiconstrained total elbow replacement in RA. Orthop Trans 1990;14:104.

45. O'Driscoll SW, Morrey BF. Coonrad-Morrey semiconstrained total elbow arthroplasty. In: Rüther $\mathrm{W}$, ed. The elbow: endoprosthetic replacement and non-endoprosthetic procedures. Berlin: Springer, 1995:111-20.
46. Souter WA. The evolution of total replacement arthroplasty of the elbow. In: Kashiwagi D, ed. Elbow joint. Proceedings of the international seminar Kobe, Japan, Amsterdam. Excerpta Medica International Congress Series, 1985;678:255-68.

47. Souter WA. Le traitement chirurgical du coude rhumatoide: Cahiers d'enseignement de la SOFCOT. Conférences d'enseignement 1987;1: 159-72.

48. Trail IA, Nuttall D, Stanley JK. Survivorship and radiological analysis of the standard Souter-Strathclyde total elbow arthroplasty. $J$ Bone Joint Surg [Br] 1999;81-B:80-4.

49. Gill DRJ, Morrey BF. The Coonrad-Morrey total elbow arthroplasty in patients who have rheumatoid arthritis: a ten to fifteen-year study. $J$ Bone Joint Surg [Am] 1998;80-A:1327-35.

50. Gschwend N. Salvage procedure in failed elbow prosthesis. Arch Orthop Trauma Surg 1983;101:95-9.

51. Gschwend $\mathbf{N}$, Loehr J, Ivosevic-Radovanovic $D$, Scheier $H$, Munzinger U. Semiconstrained elbow prosthesis with special reference to the GSB III prosthesis. Clin Orthop 1988;232:104-11.

52. Simmen BR, Gschwend N. Elbow arthroplasty - Cinderella of arthroplasty: reconstruction of the elbow with the GSB III elbow prosthesis. In: Baumgartner $\mathrm{H}$, Dvořák J, Grob D, Munziger U, Simmen B, eds. Rheumatoid arthritis: current trends in diagnostics, conservative treatment, and surgical reconstruction. Stuttgart: Thieme, 1995: 198-21. 